\title{
Known Knowns and Known Unknowns on Behavior Change Interventions
}

\section{and Mechanisms of Action}

Martin S. Hagger ${ }^{1,2}$, Susette Moyers ${ }^{1}$, Kaylyn McAnally ${ }^{1}$, and Lauren E. McKinley ${ }^{1}$

${ }^{1}$ Psychological Sciences, University of California, Merced

${ }^{2}$ Faculty of Sport and Health Sciences, University of Jyväskylä

\section{Note}

Martin Hagger's contribution was supported by a Finnish Distinguished Professor (FiDiPro) award from Business Finland (1801/31/2015). The authors declare no interests relating to this manuscript. Correspondence concerning this article should be addressed to Martin S. Hagger, SHARPP Lab,

Psychological Sciences, University of California, Merced, 5200 North Lake Road, Merced, CA 95343, USA. Email: mhagger@ucmerced.edu

Karina W. Davidson served as Editor-in-Chief for this article. 


\begin{abstract}
Systematic reviews and meta-analyses play an important role in summarizing current research on the efficacy of behavior change interventions and their mechanisms of action. The reviews in this special issue represent a 'step change' in evaluating current evidence on behavior change interventions and mechanisms. This article outlines the findings and emerging issues identified in the reviews ('known knowns'), and summarizes evidence gaps to be addressed in future research ('known unknowns'). Findings indicate that tests of mechanisms of behavior change interventions are not routinely conducted in primary studies and research syntheses; reviews and studies do not sufficiently account for study quality; substantive variability exists in descriptions of intervention content and putative mediators implicated in their mechanisms of action; limited data is available on the efficacy of many behavior change techniques; and moderators of intervention effects and mechanisms are seldom taken into account. Possible solutions include testing effects of isolated behavior change techniques and mechanisms of action; routine evaluation of study quality in behavioral intervention research; development of an evidence base linking behavior change techniques with theory-based constructs that comprise mechanisms of action; adoption of fit-for-purpose methods for synthesizing behavioral intervention mechanisms of action; and routine testing of moderators in intervention research.
\end{abstract}

Keywords: behavior change technique; mechanisms of action; behavioral theory; behavior change intervention; systematic reviews and meta-analyses; putative mediators 


\section{Introduction}

Considerable epidemiologic research has demonstrated consistent links between chronic disease risk and behaviors (Ford, Zhao, Tsai, \& Li, 2011; Li et al., 2018). Research in preventive medicine has identified behaviors that offer protection from chronic disease including 'lifestyle' behaviors like physical activity, healthy eating, not smoking, and drinking only in moderation or not at all, as well as behaviors that support early detection of risk such as cancer screening or genetic testing, or promote effective management of long-term conditions such as adherence to medication (Spring, Moller, \& Coons, 2012). Given evidence that uptake of, and adherence to, these kinds of preventive behaviors is low, policymakers and research funders have prioritized the development of effective behavioral interventions targeting population-level change in these behaviors (Glanz \& Bishop, 2010; Johnson \& Acabchuk, 2018). The vision is the generate a substantive shift in overall behavioral patterns toward levels that will confer large, lasting changes in clinically-relevant outcomes and risk of chronic disease. Such an approach is not only essential to manage the substantive human cost of chronic behaviorrelated disease and conditions, but also critical to making future healthcare costs affordable in a growing, aging global population. In this context, development of optimally efficacious, effective, costeffective, implementable, and scalable behavioral interventions presents a grand challenge to scientists in the fields of social and behavioral science and allied disciplines including public health, behavioral and preventive medicine, and health communication (Czajkowski et al., 2015; Hagger, Cameron, Hamilton, Hankonen, \& Lintunen, 2020; Rothman, Klein, \& Sheeran, 2020).

While research and implementation of behavioral interventions aimed at promoting population level participation in key behaviors has proliferated, it is only relatively recently that scientists have begun to systematically apply knowledge derived from theories from the behavioral sciences in the development of interventions and develop a science of behavior change (Aklin et al., 2020; Aunger \& Curtis, 2016; Michie \& Johnston, 2012; National Institutes of Health, 2019). Research in this emerging discipline has developed and promulgated theories and produced research evidence on how behavioral 
interventions 'work' and intervention content and methods likely to be optimally effective in evoking change across multiple behaviors, contexts, and populations (Michie \& West, 2013). Research seeking to understand the mechanisms by which behavioral interventions lead to change is critical to inform this understanding, and the role of theories applied from psychology, particularly those that focus on motivation and self-regulation, feature prominently (Glanz \& Bishop, 2010; Hagger, 2010; Hagger \& Weed, 2019; Johnson \& Acabchuk, 2018; Klein, Shepperd, Suls, Rothman, \& Croyle, 2015; Michie \& Johnston, 2012; Rothman, Klein, \& Cameron, 2013). The role of theory is critical as it provides a framework to identify potentially effective intervention content and processes by which that content is hypothesized to change behavior, which can then be systematically tested against observations, alongside any auxiliary assumption and potential moderating variables (Glanz \& Bishop, 2010; Hagger, Gucciardi, \& Chatzisarantis, 2017; Michie \& Johnston, 2012; Rothman et al., 2013; Sheeran, Klein, \& Rothman, 2017). It also permits the rejection of ideas which, in itself, is important to the iterative process of development of theories in the context of behavior change.

However, as research on theory-based behavioral interventions has proliferated, syntheses of this evidence in systematic reviews and meta-analyses have become critical in order to develop a cumulative perspective on the intervention content that is effective in changing behavior, and the strength of the evidence of theory-informed mechanisms that describe how these interventions work. Even syntheses have selection criteria and boundary conditions that narrow their focus, and drawing generalizable conclusions on effective interventions and the mechanisms involved presents considerable challenges to behavioral scientists as the evidence grows (Johnson, Scott-Sheldon, \& Carey, 2010). The current special issue of Health Psychology Review on mechanisms of health behavior change led by the Science of Behavior Change (SOBC) Research Network (Aklin et al., 2020; Davidson \& Scholz, 2020) represents a step-change in the synthesis of quality evidence on theory-informed behavior change and associated mechanisms. The approach has been to conduct an ambitious series of meta-reviews and narrative reviews summarizing findings from extant systematic reviews and meta-analyses on behavior 
change interventions and, particularly, the theory-based mechanisms involved. This approach, the first of its kind, is exemplary of a collaborative effort to synthesize current research on behavior change interventions and associated mechanisms, and provide an executive overview of the state of knowledge overall in specific fields with keen attention to key methodological artifacts that permeate the findings, such as quality of the reviews and constituent studies and the ways in which theory and intervention content has been described and coded. The research represents a considerable contribution to the field by providing the most up-to-date overview of behavior change research based on existing reviews, the caveats and limitations of these reviews, and the gaps in knowledge that warrant future research.

It is a privilege to offer a concluding perspective on the current special issue. Broadly, the current set of meta-reviews provide the most comprehensive summary data yet on the efficacy of behavior change interventions and the theory-based mechanisms of change implicated in their effects. The reviews summarize current knowledge on change interventions, and highlight the limits of that knowledge and key emerging issues. Specifically, these issues include: researchers reporting behavior change interventions do not routinely conduct tests of the mechanisms by which the interventions bring about change, and few reviewers synthesizing evidence on behavior change interventions conduct analyses testing mechanism effects; the quality of the interventions included in the reviews is suboptimal and reviews of intervention to do not sufficiently take study quality into account when assessing intervention effects; substantive variability exists in the descriptions of intervention content (e.g., techniques of behavior change interventions) and the theory-based constructs implicated in mechanisms across behavioral interventions, presenting considerable challenges to synthesis of effects and mechanisms tests across studies; strong data on key behavior change techniques is limited; and reviews tend not to sufficiently account for the effect of moderators on intervention effects and mechanisms. Here, we provide an overview of what we see are the key issues emerging from the current reviews that contribute to knowledge on behavior change mechanisms ('known knowns'), and 
identify how this knowledge signposts gaps in knowledge ('known unknowns') that need to be the targets of research endeavors by behavior change researchers going forward.

\section{Few Studies and Reviews Provide Strong Data on Behavior Change Mechanisms}

An essential consideration when it comes to evaluating behavior change interventions is a clear understanding of the link between the intervention content (usually a behavior change technique or, more commonly, a set of behavior change techniques) proposed to affect change in the behavioral outcome of an intervention, and the theory-based constructs through which the intervention content is proposed to exert its effects (Kok et al., 2016; Michie et al., 2016; Michie, Johnston, Francis, Hardeman, \& Eccles, 2008; Michie, Webb, \& Sniehotta, 2010). A priori knowledge of these links is essential for intervention designers to put into place the design features and measures required to conduct analyses to test those mechanisms. It will also assist in informing the kinds of analyses that need to be conducted are reported in order to demonstrate these effects. Such knowledge is also essential to the systematic reviewer or meta-analyst attempting to gain an estimate of the effect sizes and variability of the mechanisms of effect across the extant research by signalling the kinds of data required to provide those synthesized estimates. Knowledge of mechanisms is also key to informing the development of optimally effective interventions by providing information on the kinds of strategies and target constructs likely to be effective in changing behaviors across contexts and populations. Recognition of the importance of identifying the mechanisms responsible for effects of behavioral intervention on behavior change was a major driver of the SOBC project and a key goal of the current set of meta-reviews (Aklin et al., 2020; Davidson \& Scholz, 2020).

However, there is some misunderstanding over the key components of mechanisms of behavior change and the kinds of data required in order to provide precise evaluation of how intervention work in changing behavior. As a consequence, we provide a brief overview of the theoretical principles behind the mechanisms of effect of behavioral interventions. This is important contextual information required to provide an informed evaluation of the state of the extant research on mechanisms of behavior change 
interventions based on the reviews in the current special issue. It is also essential information required to inform future primary research and syntheses of research of the kinds of intervention content, design features, measures, and data required to provide information on mechanisms of change.

A basic process model that summarizes the key components of a mechanism of action in behavior change interventions is illustrated in Figure 1. The model outlines the key components of the mechanism, and, by implication, the types of data and analysis necessary to provide evidence to test the proposed mechanism. The model presented in Figure 1a depicts the effects of the content of an intervention - in its simplest form, an individual behavior change method or technique such as those illustrated in behavior change technique taxonomies - on change in a target behavior mediated by, for example, a single modifiable factor (e.g., a psychological construct). Specifically, the change technique is expected to affect change in the factor proposed in theory to be responsible for behavior change (the path labelled $a$ in Figure 1a). The factor is expected to affect concomitant change in the behavior (the path labelled $b$ in Figure 1a). Together these effects comprise a mediated or indirect effect which is proposed to account for the direct effect of the intervention on behavior change (represented by path $c^{\prime}$ in Figure 1a). If the modifiable factor fully accounts for the effect of the technique on behavior change, then there will be no remaining or residual effect of the technique on behavior change ${ }^{1}$. The direct effect of the technique on behavior change independent of the mediated effect represents a test of the efficacy of the intervention content in changing behavior (represented by path $c$ in Figure 1b). This is usually the focal, and often the only, effect of interest to those evaluating an intervention. Together, these effects reflect the process by which the intervention is expected to work in changing behavior consistent with theory and together represent a mechanism of action ${ }^{2}$.

\footnotetext{
${ }^{1}$ Analytically, the mechanism of action is most effectively evaluated using mediation analyses (Hayes, 2018). To the extent that the proposed mechanism of action fully accounts for the effect of the technique in changing behavior, the indirect effect represented by paths $a$ and $b$ Figure 1a should be approximately equivalent in size to the direct effect illustrated by path $c$ in Figure 1b. Interested readers are directed to Hayes' (2018) lucid treatment of this subject and accompanying process diagrams for more details.

${ }^{2}$ It is important to be note that the term mechanism of action encompasses the effect of the intervention (behavior change technique) on behavior change through the theory-based factor. Links between behavior change techniques and modifiable factors (illustrated by path $a$ in Figure 1a), and between factors and behavior change (illustrated by path $b$ in Figure 1a), represent constituent parts of the mechanism of action and not the mechanism of action itself.
} 
It is important to note that the illustration of the mechanism of action likely represents a simplified case. Many techniques are proposed to affect behavior change through change in multiple theory-related factors or mediators, so there may be other modifiable factors mediating the effect of the technique on behavior change and therefore multiple indirect effects (illustrated in Figure 1a by the $i$ suffix alongside the modifiable factor and the $a$ and $b$ paths comprising the mediated effect). To further add to the complexity, many interventions also comprise multiple techniques, each with their own mediating factor or set of factors. This presents problems in specifying and analyzing mechanisms of action. It is not possible to truly isolate the mechanisms of action of interventions comprising multiple techniques unless the intervention adopts a factorial design which enables testing proposed mechanisms for each technique in isolation. Overall, the basic process model serves a dual purpose: (i) it serves as a template for the a priori specification of the modifiable factors or constructs that are targeted in an intervention (cf., putative intervention target; Aklin et al., 2020; Hagger, 2019a; Nielsen et al., 2018), and expected mechanism by which the intervention is expected to 'work' in changing behavior, consistent with theory; and (ii) provides a template for the kinds of measures and data necessary to test the proposed mechanism of action, and the analyses required to support it.

A relatively recent contribution to knowledge of behavior change mechanisms has been the development of databases comprising expert-verified links between behavior change techniques and the constructs proposed to mediate their effects from multiple theories (Carey et al., 2019; Connell et al., 2018; Michie et al., 2017). Such information is extremely important as it provides researchers and intervention designers guidance on essential components that comprise mechanisms of action (Byrne, 2020). However, cumulative evidence from tests of mechanisms summarized in the basic process model is needed, and the current set of meta-reviews represent an important endeavor to evaluate the extent of the current research evidence on mechanisms. Such research would be expected to contribute to the iterative updating process of knowledge of the mechanisms of action of behavior change interventions. 
However, a key finding of the current meta-reviews is that strong cumulative evidence in support of behavioral intervention mechanisms of action is relatively sparse (Hennessy, Johnson, Acabchuk, McCloskey, \& Stewart-James, 2020; Suls et al., 2020; Wilson et al., 2020). Specifically, only $6 \%$ of the included reviews $(k=4)$ in the parent meta-review directly examined whether changes in self-regulation coincide with changes in behavior, that is, directly or indirectly tested the mediation effect representing the mechanism of action (Hennessy et al., 2020). Similarly, the other meta-reviews noted an absence of (Suls et al., 2020), or distinct lack of (Wilson et al., 2020), mechanism tests. Sheeran et al. (2016) provide an example of a meta-analysis that provided appropriate tests of mechanisms of action. They demonstrated across experimental studies $(k=204)$ that communications targeting change in attitudes, norms, and self-efficacy resulted in changes in health behavior through changes in intention. However, approaches to testing mechanisms such as this were rare, and included reviews were more likely to focus on testing the effect of the intervention on the theory-based constructs and behavior separately. Such tests provide evidence on mechanisms only by inference because the effects are tested independently as opposed to testing the indirect effect of the intervention on behavior through constructs in a mediation analysis.

Reasons for the lack of evidence is attributable to limitations in primary studies on which the meta-analyses included in the meta-reviews were based, as well as limitations in the meta-analyses themselves. Specifically, there were relatively few interventions that included appropriate theory-based measures as dependent variables alongside primary dependent measures of behavior change, and those that do tended not to routinely conduct the appropriate analyses to test mechanisms of action, or report sufficient data to allow secondary analyses testing mechanisms (Hennessy et al., 2020; Suls et al., 2020; Wilson et al., 2020). In many cases, studies did not clearly specify a proposed theory-based mechanism for the effect of the intervention on behavior change. It seems authors of trials of behavioral interventions tend to fixate on testing intervention efficacy, that is, the effect of the intervention on the primary outcome (path $c$ in Figure 1b). Often, mediation analyses to test mechanisms of action are 
generally treated as secondary or exploratory analyses, and, as a result, are often underpowered or poorly conceived.

The lack of appropriate data from individual interventions therefore, prevented testing of intervention mechanisms in many of the included meta-analyses. In addition, few of the included metaanalyses attempted to conduct appropriate analyses on the primary data to test mechanisms of action. This precluded drawing conclusions on presumed mechanisms of action based on the cumulative data on behavioral interventions. Suls et al. (2020) provide an apt summary of the available data on tests of mechanisms of action: “...researchers appear to not be assessing how a successful intervention changes behavior (i.e., the MoA [Mechanisms of Action])" (p. 26), and offer this unedifying coda on the state of the research syntheses on behavioral intervention mechanisms: “Two decades ago, Dusseldorp, van Elderen, Maes, Meulman, and Kraaij (1999) published a meta-analysis of studies testing the efficacy of interventions for CVD prevention and management, and made this sobering observation '...in most studies, programmes are described only vaguely, without explicit reference to a theoretical model or to empirical findings supportive of specific causal relationships between a given strategy or intervention and positive effects on outcome or intermediate indicators of success. (p. 533)'. We have to conclude that progress since 1999 seems modest." (p. 30).

So, what research is needed to test mechanisms of action going forward? Primary research needs to provide clear a priori specification of the theory based mechanisms of action by which interventions are proposed to affect behavior change. Such a specification is necessary to inform the design of the intervention so that the appropriate design features, measures, and analyses will be in place to enable subsequent tests of those mechanisms of action. This means that researchers need to pay due diligence in identifying appropriate mechanisms of action based on theory and currenty available evidence, and design their study accordingly. In addition, appropriate mediation analyses and reporting of data is required in order to facilitate future research syntheses of tests of the mechanisms, preferably with open and transparent reporting of data and analyses (Hagger, 2019b; O'Connor, 2020). This means that 
mediation analyses testing mechanisms of action need to be at the forefront of analyses, rather than secondary or exploratory analyses. In addition, systematic reviews and meta-analyses need to extract and code the appropriate data necessary to conduct appropriate analyses to test mechanisms of action. Specifically, this includes appropriate coding of behavior change techniques and theory-based constructs (mediators) to ensure intervention content is appropriately pooled for analysis (Michie \& Prestwich, 2010; Michie et al., 2013; Michie, West, Sheals, \& Godinho, 2018). Appropriate analyses also need to be conducted to test the mediation effects, such as meta-analytic structural equation modeling (for applications see Cheung \& Hong, 2017; Hagger, Chan, Protogerou, \& Chatzisarantis, 2016; Hagger, Polet, \& Lintunen, 2018; Protogerou, Johnson, \& Hagger, 2018; Zhang, Zhang, Schwarzer, \& Hagger, 2019), which accounts for the indirect effects of interventions consistent with the process model outlined previously (Figure 1). For example, Rhodes, Boudreau, Weman Josefsson, and Ivarsson (2020) conducted a systematic review of theory-based physical activity interventions reporting each component effect of the basic process model. This enabled tests of the mechanisms of action by specifying mediation of intervention effects on behavior through theory-based mediators. Meta-analytic structural equation models provided support for the indirect effects of interventions on physical activity through the nominated theory-based mediators using data from multiple studies. This analysis provides a template for future tests of mechanisms of action.

A key barrier to the latter analyses is that many interventions encompass multiple intervention techniques. This creates problems isolating effects of individual behavior change techniques and the theory-based constructs implicated in the mechanism. As Suls et al. (2020) suggest, "the continuing practice of testing multicomponent behavioural interventions in aggregate" (p. 29) hinders progress in testing mechanisms of action. For example, an intervention seeking to promote physical activity may comprise persuasive communications targeting attitude change, experiences of success targeting selfefficacy change, and action planning targeting planning change simultaneously. This intervention is offered to an intervention groups and tested for efficacy in changing physical activity behavior at 
follow-up against a control or comparison group that does not receive the intervention. Such efficacy tests are silent on which of the specific techniques is causing behavior change, and whether they act independently or interactively to produce the change. Similarly, such designs preclude meaningful tests of the mechanisms of action of the intervention; even if relevant theory-based mediators had been measured, the presence of multiple techniques in a single intervention prevents mediation analyses that test the mechanisms of action of each technique independently. Solutions to these issues lie in testing interventions that test effects of individual techniques in isolation, or the use of factorial designs which permit tests of the independent and interactive effects of individual techniques, as advocated by review authors and commentators in the current special issue and elsewhere (Byrne, 2020; Peters, de Bruin, \& Crutzen, 2015; Suls et al., 2020; Wilson et al., 2020). But theses designs should also coincide with tests of mechanisms to explore the specific mechanisms by which isolated techniques exert their effect on behavior change. Such designs would accelerate the endeavors of scientists conducting reviews to synthesize effectiveness of individual behavior change techniques and their mechanisms of action, and provide valuable contributions to the fledgling evidence base of mechanisms of action (Carey et al., 2019; Connell et al., 2018). However, large-scale trials adopting such designs, especially those with multiple intervention groups, are costly, and researchers are often reluctant to design trials using isolated techniques in fear of returning null findings. Investment in such designs, is, however, very important if a comprehensive evidence base of mechanisms of action is to be built. Smaller-scale pilot and experimental trials may represent an opportunity to establish initial formative evidence for mechanisms of action. Lobbying research funders to invest in such trials using isolated techniques and factorial designs would also be an important step toward developing the mechanisms of action evidence base.

\section{Study and Review Quality}

An important theme running through the set of meta-reviews in the current special issue is the issue of quality, that is, the quality of primary studies included in systematic reviews and meta-analyses, 
and the assessment thereof, and the quality of the reviews themselves (Hennessy et al., 2020; Protogerou, McHugh, \& Johnson, 2020; Suls et al., 2020; Wilson et al., 2020). The issue of quality of the evidence included in systematic reviews and meta-analyses has been acknowledged for some time (Armijo-Olivo, Stiles, Hagen, Biondo, \& Cummings, 2012; Johnson, Low, \& MacDonald, 2014; Moyer \& Finney, 2005; Zeng et al., 2015), and guidelines exist advocating assessment of study quality and taking quality into account when providing evaluations (Cooper, 2017; Moher, Liberati, Tetzlaff, Altman, \& the PRISMA Group, 2009; Shea et al., 2017), including formal moderator analyses to establish the extent to which study quality affects conclusions (Johnson et al., 2014). In the context of behavioral interventions, it is therefore important that reviewers adopt appropriate means to evaluate study quality and conduct analyses to test for effects of study quality. In the context of tests of mechanisms of action, is important that study quality assessments adequately account for salient aspects of study design, such as use of theory and specification of mechanisms a priori, inclusion of appropriate measures, conduct of appropriate analyses (e.g., mediation analyses), and transparent reporting. Such analyses will advance knowledge by demonstrating the extent to which elements of study design affect the quality of tests of mechanisms of action. It is also important that researchers conducting metaanalyses of behavioral interventions testing mechanisms also follow quality guidelines. Reviews need to consider adhering to recommended guidelines for reviews on quality, such as the AMSTAR-2 guidelines (Shea et al., 2017). Results from the current meta-reviews suggest that adoption of these guidelines is typically low, and there is a need to promote better awareness and application of guidelines to promote higher quality review evidence on behavior change (O'Carroll, 2020).

Solutions lie in stronger advocacy of 'good practice' in assessing and reporting study quality. For example, study quality guidelines and how to apply them should become integral to training programs for doctoral students. It is also important that principal investigators leading reviews of evidence on behavior change interventions provide appropriate training on quality assessment of included studies and on components of quality for conducting reviews. Such advocacy will improve the 
overall the standards of published research and research syntheses, with a view to providing high quality tests of mechanisms in behavioral intervention. It is also important that the methods used to assess the quality of studies used in syntheses of evidence on behavior change interventions and their mechanisms are appropriate and fit-for-purpose. While standards have been set on the conduct of evidence syntheses resulting in the generation of guidelines and checklists (e.g., AMSTAR-2, PRISMA, MARS), there is, to date, no clear standard set on methods used to evaluate study quality. Researchers conducting systematic reviews and meta-analyses have tended to use bespoke tools or checklists for assessing study quality, which vary in the quality criteria they adopt and the scoring system used to produce quality scores. There have been calls for standardized methods of quality assessment (Protogerou \& Hagger, 2019), and suggestions to move away from using aggregate quality scores derived from different criteria, and instead use assessments of individual criteria as independent moderators (Johnson et al., 2014). Consensus on the section of study quality criteria to assess therefore because important in this regard. For example, assessment of the quality of the techniques used and reported in behavior change interventions is likely to be a priority consideration in evidence syntheses of these interventions (de Bruin et al., 2020). Such considerations need to be accounted for in the development of standardized methods of quality assessment specifically for behavior change interventions and will advance current methods of study quality assessment and evaluation in evidence syntheses.

\section{Clarity in Definitions of Theory Constructs and Mechanisms of Action}

A prominent theme of the current meta-reviews is the variability in definitions and operationalization of the theory-based constructs implicated in the mechanisms by which techniques from intervention impact Behavior. This should come as no surprise to psychologists, and those from the other social and behavioral sciences that inform theoretical mechanisms of behavior change interventions, given the substantive number of theories and constructs available (Fishbein et al., 2001; McMillan \& Conner, 2007; Protogerou et al., 2018). Psychology, for example, is an extremely theory 
'rich' discipline, a phenomenon that poses considerable challenges to those attempting to make sense and synthesize findings across studies. The large number of theories mean that there are many theory based constructs with similar content but named differently (a 'jangle’ fallacy, or déjà-variable problem), and also constructs with different content that have the same label (a 'jingle' fallacy), both of which impede scientific progress by masking potential trends across studies (Block, 1995; Hagger, 2014). Similarly, descriptions of behavior change techniques vary considerably across studies in terms of the labels used and detail provided. The variability in definitions and descriptions make it difficult for researchers synthesise findings on behavior change interventions and their associated mechanisms of action across studies (Abraham \& Michie, 2008; Michie \& Abraham, 2008; Michie \& Johnston, 2012).

Solutions lie in the development of common sets of terms and language use to describe the content of interventions and the constructs used. Behavior change technique classifications and taxonomies have done much to provide a common set of descriptions for techniques used in behavior change interventions (e.g., Abraham \& Michie, 2008; Hardcastle, Fortier, Blake, \& Hagger, 2017; Knittle et al., 2020; Kok et al., 2016; Michie et al., 2013; Teixeira et al., 2020). However, intervention designers have not readily adopted these common descriptions when specifying their interventions (Hennessy et al., 2020; Suls et al., 2020), which necessitates the development and application of coding systems to 'translate' descriptions of interventions into a common vernacular that enables synthesis (e.g., Black et al., 2018; Gilinsky et al., 2015). The behavior change technique taxonomies and associated coding schemes have been used to some extent in the reviews included in the current metareviews, and have enabled some tests of efficacy of intervention techniques across studies, albeit with the caveats outlined earlier. Recent work aimed at linking theoretical constructs with behavior change techniques has aimed to achieve similar common descriptions for theoretical constructs and their associated techniques (Carey et al., 2019; Connell et al., 2018; Kok et al., 2016). The goal is to develop a database of mechanisms of action that can be used to provide better descriptions of intervention 
mechanism in primary research, and synthesis of findings on intervention mechanisms across studies. However, such work is relatively new and, as findings of current set of meta-reviews attest, has yet to be applied in the descriptions of behavior change interventions mechanisms in research syntheses. Future systematic reviews and meta-analyses aimed at estimating effect sizes for tests of theory-based intervention mechanisms needs to apply such coding systems, similar to those used by Rhodes et al. (2020). In addition, researchers also need to become more systematic in specifying the theory-based mechanisms through which they expect their behavior change interventions to work. This necessitates clear specification of each component of the intervention using the basic model of behavior change mechanisms as a template. Existing (Bartholomew Eldredge et al., 2016; Hayes, 2018 ) and newly developed (West et al., 2019) systems for specifying links between techniques, constructs, and behavior that form mechanisms of action can facilitate this specification, although these should not be merely descriptive, but should also have utility in, for example, linking the model to the analyses necessary to test them.

\section{Limited Strong Data on Efficacy and Mechanisms of Action for Many Behavior Change}

\section{Techniques}

The 'parent' meta-review in the current special issue identified 17 behavior change techniques that featured prominently in the behavioral interventions reported in the included reviews (Hennessy et al., 2020), and some of the associated reviews focused on smaller sets of techniques (Suls et al., 2020; Wilson et al., 2020). However, few of the included reviews conducted direct tests of the effect of the intervention on constructs implicated in the proposed change mechanisms alongside changes in behavior, and fewer still tested the indirect effect (e.g., Protogerou et al., 2020; Suls et al., 2020; Wilson et al., 2020). Those that did focused on a narrow set of techniques including those promoting selfefficacy, frequency of self-monitoring, and cognitive bias training (e.g., Sheeran et al., 2016). In addition, evidence for intervention efficacy and effects on constructs representing change mechanisms for many of the techniques in the identified set was very limited, including self-affirmation, self- 
management, stress-management, time-management, implementation-intention interventions, self-talk, inhibitory/self-control training, relapse prevention/coping planning, reviewing goals, action-planning, and barrier identification and problem solving techniques (Hennessy et al., 2020). So, while data from primary studies appear to demonstrate the potential efficacy of these techniques in changing behavior, meta-reviews indicated substantive variability in effect sizes of efficacy tests across studies, and the quality of studies is low. Furthermore, many research syntheses use presence vs. absence analyses in meta-regression to evaluate efficacy of these techniques, which has inherent limitations due to variability in the presence of other potentially confounding or interacting techniques (Peters et al., 2015), and the presence of other techniques that co-occur across intervention and control groups that need to be coded or controlled for (de Bruin et al., 2020).

Resolving these problems requires more systematic primary tests of efficacy of isolated behavior change techniques on behavior, or factorial designs that test main and interactive effects of isolated techniques, as called for by authors of the current meta-reviews and outlined in the previous section (Hennessy et al., 2020; Suls et al., 2020). A similar approach needs to be taken to the testing of the mechanisms of action involved, including the appropriate design features, measures, and analyses to do so. In addition, those conducting systematic reviews and meta-analyses need to also become more cognizant of the necessity of extracting appropriate data on mechanisms of action (e.g., tests of the paths proposed in the basic model of mechanisms of action, Figure 1), and the types of analyses required to appropriately test these mechanisms (e.g., meta-analytic structural equation modeling). Some reviews provide a blueprint for such analyses, such as those by Sheeran et al. (2016) and Rhodes et al. (2020). These studies represent the progressive designs necessary to provide syntheses of research on mechanisms of action, and the current meta-reviews have highlighted that reviews adopting such designs are rare.

\section{Reviews and Meta-Analyses Do Not Routinely Account for Key Moderators.}


A noted deficiency of the systematic reviews and meta-analyses included in the current metareviews is the lack of consideration of moderators of the both the efficacy of interventions in changing behavior and the mechanisms involved (Byrne, 2020). Key moderators flagged in the current set of reviews include social environmental factors and methodological artifacts. Alcántara et al.’s (2020) review, in particular, suggested that systematic reviews and meta analyses of behavior change interventions generally tended to focus on a narrow set of moderators of intervention effects including individual demographic factors like gender and ethnicity/race, but not occupation and unemployment status, and intervention location. However, few accounted for socio-ecological and structural factors such as neighbourhood characteristics (e.g., levels of crime and violence, environmental presence of health-promoting factors), rural or urban setting, access to healthcare, and exposure to discrimination/racism. Similarly, Miller et al. (2020) indicate the need to account for developmental factors when developing behavior change intervention given age-related changes in self-regulatory capacity that occur in childhood through adolescence and into adulthood. Finally, behavior type is a potentially important moderator of behavioral intervention effects, but studies seldom provide the necessary summary data to conduct a moderator analysis by behavior type (Hennessy et al., 2020). Evidence for the effects of these factors on effectiveness of interventions is lacking, particularly effects of social environmental factors. Reviews also flagged the importance of methodological moderators of behavior change intervention effects, particularly method of delivery (see Dombrowski, O'Carroll, \& Williams, 2016; Hagger \& Hardcastle, 2014; O'Carroll, 2020), intervention 'dose', and experience and training of those administering the intervention, but the reviews indicated virtually no systematic treatment of these moderators in the included reviews (Hennessy et al., 2020; Wilson et al., 2020).

Solutions are the identification, extraction, and coding of key moderator variables as a matter of course when conducting systematic reviews and meta-analyses of behavior change interventions. In the context of a large, expanding, and increasingly complex literature on behavior change interventions, the application of automated search methods may facilitate these processes (Marshall, Johnson, Wang, 
Rajasekaran, \& Wallace, 2020). Such endeavors will facilitate exploration of the effects of moderators by comparing behavior change intervention effects of behaviors across levels of the moderator in metaanalyses. These analyses, however, are only feasible if primary research routinely includes suitable measures and tests of moderator effects, and report sufficient data to permit estimation of effect sizes. Primary studies examining effects of behavior change interventions in minority groups and populations with health disparities are needed (Alcántara et al., 2020). In addition, studies that test intervention efficacy and mechanisms of action when other methodological artifacts like delivery mode, intervention dose, and training level of delivery staff are varied are also needed. Such analyses will be facilitated by appropriate guidelines and checklists for researchers that advocate moderator tests, and journal editors encouraging, rewarding, or even formally requiring moderator analyses in syntheses of research on behavioral interventions. Such cultural changes in the approach to behavior change intervention research are likely to be slow, but necessary, if an evidence base of conditional factors that magnify or diminish the efficacy of behavioral interventions, and their associated mechanisms of action, is to be developed.

\section{Conclusion}

The reviews reported in the current special issue provide the most comprehensive compendium available of current knowledge on behavior change interventions and their associated mechanisms of action. Going forward, we expect the reviews to serve as an essential, go-to resource for researchers, reviewers, and interventionists with a vested interest in developing and evaluating behavior change interventions. In this commentary we have highlighted how the current set of reviews make an original contribution furthering understanding of interventions and mechanisms; substantiating the 'known knowns' in the field. These include identification of specific techniques known to be efficacious in bringing about behavior change broadly across studies on behavior change interventions, the extent to which coding schemes and meta-regression analyses are used to test efficacy of specific behavior change techniques, the methods researchers have used in systematic reviews and meta-analyses to 
evaluate or formally test the mechanisms of action by which intervention content 'works' in changing behavior, and the methods used to evaluate the quality of behavior change intervention research.

More poignantly, the current reviews also signpost the evidence gaps in, and the limitations and deficiencies of, extant research on behavior change intervention efficacy and mechanisms - knowledge we do not know, 'known unknowns', and need to know in order to move the field of toward the development of a comprehensive evidence base of optimally-efficacious behavior change interventions and their mechanisms of action. These include the lack of strong evidence of the efficacy of many specific behavior change techniques and their mechanisms of action, limitations in the assessment of study quality, the lack of clarity in definitions and descriptions of constructs and techniques, and the need to routinely include measures and tests of key individual, social environmental, and methodological moderators of intervention effectiveness.

Here, we have highlighted some potential solutions to fill these knowledge gaps derived from the meta-reviews and commentaries and further afield. In particular, we advocate (a) testing effects of behavior change techniques in isolation and adoption of factorial designs to test independent and interactive effects of techniques; (b) advocacy of 'good practice' guidelines for study quality evaluation in behavioral intervention research, and standardized tools to evaluate intervention quality; (c) continued development of databases of behavior change techniques and links with theoretical constructs involved in mechanisms of action; (d) conducting systematic reviews and meta-analyses that adopt fitfor-purpose methods for synthesizing studies testing intervention mechanisms of action; and (e) routine inclusion and coding of appropriate measures of moderators in intervention research and research syntheses. Multiple programs of research from teams of researchers is required to systematically address each of these proposed solutions, and funders should be lobbied to resource the programs. The current funded line of research is a fitting exemplar of what can be achieved through targeted, sustained investment in behavioral solutions to current health problems. 


\section{References}

Abraham, C., \& Michie, S. (2008). A taxonomy of behavior change techniques used in interventions. Health Psychology, 27, 379-387. https://doi.org/10.1037/0279-6133.27.3.379

Aklin, W. M., Stoeckel, L., Green, P., Keller, C., King, J. W., Nielsen, L., \& Hunter, C. (2020). Commentary: National Institutes of Health (NIH) Science of Behavior Change (SOBC). Health Psychology Review. https://doi.org/10.1080/17437199.2020.1716383

Alcántara, C., Diaz, S. V., Cosenzo, L. G., Loucks, E. B., Penedo, F. J., \& Williams, N. J. (2020). Social determinants as moderators of health behaviour change interventions: Scientific gaps and opportunities. Health Psychology Review. https://doi.org/10.1080/17437199.2020.1718527

Armijo-Olivo, S., Stiles, C. R., Hagen, N. A., Biondo, P. D., \& Cummings, G. G. (2012). Assessment of study quality for systematic reviews: A comparison of the Cochrane Collaboration Risk of Bias Tool and the Effective Public Health Practice Project Quality Assessment Tool: methodological research. Journal of Evaluation in Clinical Practice, 18, 12-18. https://doi.org/doi:10.1111/j.1365-2753.2010.01516.x

Aunger, R., \& Curtis, V. (2016). Behaviour centred design: Towards an applied science of behaviour change. Health Psychology Review, 10, 425-446. https://doi.org/10.1080/17437199.2016.1219673

Bartholomew Eldredge, L. K., Markham, C. M., Ruiter, R. A. C., Fernández, M. E., Parcel, G. S., \& Kok, G. (2016). Planning health promotion programs: An intervention mapping approach (4th ed.). San Francisco, CA: Jossey-Bass.

Black, N., Williams, A. J., Javornik, N., Scott, C., Johnston, M., Eisma, M. C., . . de Bruin, M. (2018). Enhancing behavior change technique coding methods: Identifying behavioral targets and delivery styles in smoking cessation trials. Annals of Behavioral Medicine, 53, 583-591. https://doi.org/10.1093/abm/kay068

Block, J. (1995). A contrarian view of the five-factor approach to personality description. Psychological Bulletin, 117, 187-215. https://doi.org/10.1037/0033-2909.117.2.187 
Byrne, M. (2020). Gaps and priorities in advancing methods for health behaviour change research. Health Psychology Review. https://doi.org/10.1080/17437199.2019.1707106

Carey, R. N., Connell, L. E., Johnston, M., Rothman, A. J., de Bruin, M., Kelly, M. P., \& Michie, S. (2019). Behavior change techniques and their mechanisms of action: A synthesis of links described in published intervention literature. Annals of Behavioral Medicine, 53, 693-707. https://doi.org/10.1093/abm/kay078

Cheung, M. W. L., \& Hong, R. Y. (2017). Applications of meta-analytic structural equation modeling in health psychology: Examples, issues, and recommendations. Health Psychology Review, 11, 265-279. https://doi.org/10.1080/17437199.2017.1343678

Connell, L. E., Carey, R. N., de Bruin, M., Rothman, A. J., Johnston, M., Kelly, M. P., \& Michie, S. (2018). Links between behavior change techniques and mechanisms of action: An expert consensus study. Annals of Behavioral Medicine, 53, 708-720. https://doi.org/10.1093/abm/kay082

Cooper, H. (2017). Research synthesis and meta-analysis: A step-by-step approach (5th ed.). Thousand Oaks, CA: Sage.

Czajkowski, S. M., Powell, L. H., Adler, N., Naar-King, S., Reynolds, K. D., Hunter, C. M., .. . Charlson, M. E. (2015). From ideas to efficacy: The ORBIT model for developing behavioral treatments for chronic diseases. Health Psychology, 34, 971-982. https://doi.org/10.1037/hea0000161

Davidson, K. W., \& Scholz, U. (2020). Understanding and predicting health behavior change: A contemporary view through the lenses of meta-reviews. Health Psychology Review. https://doi.org/10.1080/17437199.2020.1719368

de Bruin, M., Black, N., Javornik, N., Viechtbauer, W., Eisma, M., Hartmann-Boyce, J., . . Johnston, M. (2020). Underreporting of the active content of behavioural interventions: A systematic review 
and meta-analysis of randomized trials of smoking cessation interventions. Health Psychology Review. https://doi.org/10.1080/17437199.2019.1709098

Dombrowski, S. U., O'Carroll, R. E., \& Williams, B. (2016). Form of delivery as a key 'active ingredient' in behaviour change interventions. British Journal of Health Psychology, 21, 733-740. https://doi.org/10.1111/bjhp.12203

Dusseldorp, E., van Elderen, T., Maes, S., Meulman, J., \& Kraaij, V. (1999). A meta-analysis of psychoeducational programs for coronary heart disease patients. Health Psychology, 18, 506-519. https://doi.org/10.1037/0278-6133.18.5.506

Fishbein, M., Triandis, H. C., Kanfer, F. H., Becker, M., Middlestadt, S. E., \& Eichler, A. (2001). Factors influencing behavior and behavior change. In A. Baum, T. A. Revenson \& J. E. Singer (Eds.), Handbook of Health Psychology (pp. 3-17). Mahwah, NJ: Lawrence Erlbaum.

Ford, E. S., Zhao, G. X., Tsai, J., \& Li, C. Y. (2011). Low-risk lifestyle behaviors and all-cause mortality: Findings from the national health and nutrition examination survey III mortality study. American Journal of Public Health, 101, 1922-1929. https://doi.org/10.2105/ajph.2011.300167

Gilinsky, A. S., Dale, H., Robinson, C., Hughes, A. R., McInnes, R., \& Lavallee, D. (2015). Efficacy of physical activity interventions in post-natal populations: Systematic review, meta-analysis and content coding of behaviour change techniques. Health Psychology Review, 9, 244-263. https://doi.org/10.1080/17437199.2014.899059

Glanz, K., \& Bishop, D. B. (2010). The role of behavioral science theory in development and implementation of public health interventions. Annual Review of Public Health, 31, 399-418. https://doi.org/10.1146/annurev.publhealth.012809.103604

Hagger, M. S. (2010). Self-regulation: An important construct in health psychology research and practice. Health Psychology Review, 4, 57-65. https://doi.org/10.1080/17437199.2010.503594

Hagger, M. S. (2014). Avoiding the 'déjà-variable' phenomenon: Social psychology needs more guides to constructs. Frontiers in Psychology, 5, 52. https://doi.org/10.3389/fpsyg.2014.00052 
Hagger, M. S. (2019a). Basic model of a behavior change mechanism of action. PsyArXiv. https://doi.org/10.31234/osf.io/9a5k6

Hagger, M. S. (2019b). Embracing open science and transparency in health psychology. Health Psychology Review, 13, 131-136. https://doi.org/10.1080/17437199.2019.1605614

Hagger, M. S., Cameron, L. D., Hamilton, K., Hankonen, N., \& Lintunen, T. (2020). The science of behavior change: The road ahead. In M. S. Hagger, L. D. Cameron, K. Hamilton, N. Hankonen \& T. Lintunen (Eds.), The Handbook of Behavior Change. New York, NY: Cambridge University Press.

Hagger, M. S., Chan, D. K. C., Protogerou, C., \& Chatzisarantis, N. L. D. (2016). Using meta-analytic path analysis to test theoretical predictions in health behavior: An illustration based on metaanalyses of the theory of planned behavior. Preventive Medicine, 89, 154-161. https://doi.org/10.1016/j.ypmed.2016.05.020

Hagger, M. S., Gucciardi, D. F., \& Chatzisarantis, N. L. D. (2017). On nomological validity and auxiliary assumptions: The importance of simultaneously testing effects in social cognitive theories applied to health behavior and some guidelines. Frontiers in Psychology, 8, 1933. https://doi.org/10.3389/fpsyg.2017.01933

Hagger, M. S., \& Hardcastle, S. J. (2014). Interpersonal style should be included in taxonomies of behaviour change techniques. Frontiers in Psychology, 5, 254. https://doi.org/10.3389/fpsyg.2014.00254

Hagger, M. S., Polet, J., \& Lintunen, T. (2018). The reasoned action approach applied to health behavior: Role of past behavior and test of some key moderators using meta-analytic structural equation modeling. Social Science \& Medicine, 213, 85-94.

https://doi.org/10.1016/j.socscimed.2018.07.038 
Hagger, M. S., \& Weed, M. E. (2019). DEBATE: Do behavioral interventions work in the real world? International Journal of Behavioral Nutrition and Physical Activity, 16, 36. https://doi.org/10.1186/s12966-019-0795-4

Hardcastle, S. J., Fortier, M. S., Blake, N., \& Hagger, M. S. (2017). Identifying content-based and relational techniques to change behavior in motivational interviewing. Health Psychology Review, 11, 1-16. https://doi.org/10.1080/17437199.2016.1190659

Hayes, A. F. (2018). Introduction to mediation, moderation, and conditional process analysis: A regression-based approach (2nd ed.). New York, NY: Guildford Press.

Hennessy, E. A., Johnson, B. T., Acabchuk, R. L., McCloskey, K., \& Stewart-James, J. (2020). Selfregulation mechanisms in health behaviour change: A systematic meta-review of meta-analyses, 2006-2017. Health Psychology Review. https://doi.org/10.1080/17437199.2019.1679654

Johnson, B. T., \& Acabchuk, R. L. (2018). What are the keys to a longer, happier life? Answers from five decades of health psychology research. Social Science \& Medicine, 196, 218-226. https://doi.org/10.1016/j.socscimed.2017.11.001

Johnson, B. T., Low, R. E., \& MacDonald, H. V. (2014). Panning for the gold in health research: Incorporating studies' methodological quality in meta-analysis. Psychology \& Health, 30, 135152. https://doi.org/10.1080/08870446.2014.953533

Johnson, B. T., Scott-Sheldon, L. A. J., \& Carey, M. P. (2010). Meta-synthesis of health behavior change meta-analyses. American Journal of Public Health, 100, 2193-2198. https://doi.org/10.2105/ajph.2008.155200

Klein, W. M. P., Shepperd, J. A., Suls, J., Rothman, A. J., \& Croyle, R. T. (2015). Realizing the promise of social psychology in improving public health. Personality and Social Psychology Review, 19, 77-92. https://doi.org/10.1177/1088868314539852 
Knittle, K., Heino, M. T. J., Marques, M. M., Stenius, M., Beattie, M., Ehbrecht, F., . . Hankonen, N. (2020). The compendium of self-enactable techniques to change and self-manage motivation and behaviour v1. 0. Nature Human Behavior. https://doi.org/10.1038/s41562-019-0798-9

Kok, G., Gottlieb, N. H., Peters, G.-J. Y., Mullen, P. D., Parcel, G. S., Ruiter, R. A. C., . .

Bartholomew, L. K. (2016). A taxonomy of behavior change methods: An intervention mapping approach. Health Psychology Review, 10, 297-312. https://doi.org/10.1080/17437199.2015.1077155

Li, Y., Pan, A., Wang, D. D., Liu, X., Dhana, K., Franco, O. H., . . Hu, F. B. (2018). Impact of healthy lifestyle factors on life expectancies in the US population. Circulation. https://doi.org/10.1161/CIRCULATIONAHA.117.032047

Marshall, I. J., Johnson, B. T., Wang, Z., Rajasekaran, S., \& Wallace, B. C. (2020). Semi-automated evidence synthesis in health psychology: Current methods and future prospects. Health Psychology Review. https://doi.org/10.1080/17437199.2020.1716198

McMillan, B., \& Conner, M. (2007). Health cognition assessment. In A. B. S. Ayers, C. McManus, S. Newman, K. Wallston, J. Weinman, \& R. West (Ed.), Cambridge Handbook of Psychology, Health and Medicine (2nd ed., pp. 260-266). Cambridge, UK: Cambridge University Press.

Michie, S., \& Abraham, C. (2008). Advancing the science of behaviour change: A plea for scientific reporting. Addiction, 103, 1409-1410. https://doi.org/10.1111/j.1360-0443.2008.02291.x

Michie, S., Carey, R., Johnston, M., Rothman, A. J., Kelly, M., Davidson, K., \& de Bruin, M. (2016). From theory-inspired to theory-based interventions: Linking behaviour change techniques to their mechanisms of action. European Health Psychologist, 18, 395.

Michie, S., Carey, R. N., Johnston, M., Rothman, A. J., de Bruin, M., Kelly, M. P., \& Connell, L. E. (2017). From theory-inspired to theory-based interventions: A protocol for developing and testing a methodology for linking behaviour change techniques to theoretical mechanisms of action. Annals of Behavioral Medicine, 52, 501-512. https://doi.org/10.1007/s12160-016-9816-6 
Michie, S., \& Johnston, M. (2012). Theories and techniques of behaviour change: Developing a cumulative science of behaviour change. Health Psychology Review, 6, 1-6. https://doi.org/10.1080/17437199.2012.654964

Michie, S., Johnston, M., Francis, J., Hardeman, W., \& Eccles, M. (2008). From theory to intervention: Mapping theoretically derived behavioural determinants to behaviour change techniques. Applied Psychology-an International Review-Psychologie Appliquee-Revue Internationale, 57, 660-680. https://doi.org/10.1111/j.1464-0597.2008.00341.x

Michie, S., \& Prestwich, A. (2010). Are interventions theory-based? Development of a theory coding scheme. Health Psychology, 29, 1-8. https://doi.org/10.1037/a0016939

Michie, S., Richardson, M., Johnston, M., Abraham, C., Francis, J., Hardeman, W., .. . Wood, C. E. (2013). The Behavior Change Technique Taxonomy (v1) of 93 Hierarchically Clustered Techniques: Building an International Consensus for the Reporting of Behavior Change Interventions. Annals of Behavioral Medicine, 46, 81-95. https://doi.org/10.1007/s12160-013$\underline{9486-6}$

Michie, S., Webb, T. L., \& Sniehotta, F. F. (2010). The importance of making explicit links between theoretical constructs and behaviour change techniques. Addiction, 105, 1897-1898. https://doi.org/10.1111/j.1360-0443.2010.03161.x

Michie, S., \& West, R. (2013). Behaviour change theory and evidence: A presentation to Government. Health Psychology Review, 7, 1-22. https://doi.org/10.1080/17437199.2011.649445

Michie, S., West, R., Sheals, K., \& Godinho, C. A. (2018). Evaluating the effectiveness of behavior change techniques in health-related behavior: A scoping review of methods used. Translational Behavioral Medicine, 8, 212-224. https://doi.org/10.1093/tbm/ibx019

Miller, A. L., Lo, S., Bauer, K. W., \& Fredericks, E. M. (2020). Developmentally informed behaviour change techniques to enhance self-regulation in a health promotion context: A conceptual review. Health Psychology Review. https://doi.org/10.1080/17437199.2020.1718530 
Moher, D., Liberati, A., Tetzlaff, J., Altman, D. G., \& the PRISMA Group. (2009). Preferred reporting items for systematic reviews and meta-analyses: The PRISMA statement. PLOS Med, 6, e1000097. https://doi.org/10.1371/journal.pmed.1000097

Moyer, A., \& Finney, J. W. (2005). Rating methodological quality: Toward improved assessment and investigation. Accountability in Research, 12, 299-313. https://doi.org/10.1080/08989620500440287

National Institutes of Health. (2019). Science of behavior change. Retrieved July 23, 2019, from https://scienceofbehaviorchange.org/

Nielsen, L., Riddle, M., King, J. W., Aklin, W. M., Chen, W., Clark, D., . . Weber, W. (2018). The NIH Science of Behavior Change Program: Transforming the science through a focus on mechanisms of change. Behaviour Research and Therapy, 101, 3-11. https://doi.org/10.1016/j.brat.2017.07.002

O'Carroll, R. E. (2020). Self-regulation interventions - What do we know and where should we go? Health Psychology Review. https://doi.org/10.1080/17437199.2019.1709529

O'Connor, D. B. (2020). The future of health behaviour change interventions: Opportunities for open science and personality research. Health Psychology Review. https://doi.org/10.1080/17437199.2019.1707107

Peters, G.-J. Y., de Bruin, M., \& Crutzen, R. (2015). Everything should be as simple as possible, but no simpler: Towards a protocol for accumulating evidence regarding the active content of health behaviour change interventions. Health Psychology Review, 9, 1-14. https://doi.org/10.1080/17437199.2013.848409

Protogerou, C., \& Hagger, M. S. (2019). A case for a study quality appraisal in survey studies in psychology. Frontiers in Psychology, 9, 2788. https://doi.org/10.3389/fpsyg.2018.02788 
Protogerou, C., Johnson, B. T., \& Hagger, M. S. (2018). An integrated model of condom use in subSaharan African youth: A meta-analysis. Health Psychology, 37, 586-602. https://doi.org/10.1037/hea0000604

Protogerou, C., McHugh, R. K., \& Johnson, B. T. (2020). How best to reduce unhealthy risk-taking behaviours? A meta-review of evidence syntheses focused on self-regulation interventions. Health Psychology Review. https://doi.org/10.1080/17437199.2019.1707104

Rhodes, R. E., Boudreau, F., Weman Josefsson, K., \& Ivarsson, A. (2020). Mediators of physical activity behavior change interventions among adults: A systematic review and meta-analysis. Health Psychology Review. https://doi.org/10.1080/17437199.2019.1706614

Rothman, A. J., Klein, W. M. P., \& Cameron, L. D. (2013). Advancing innovations in social/personality psychology and health: Opportunities and challenges. Health Psychology, 32, 602-608. https://doi.org/10.1037/a0032116

Rothman, A. J., Klein, W. M. P., \& Sheeran, P. (2020). Moving from theoretical principles to intervention strategies: Applying the experimental medicine approach. In M. S. Hagger, L. D. Cameron, K. Hamilton, N. Hankonen \& T. Lintunen (Eds.), The Handbook of Behavior Change. New York, NY: Cambridge University Press.

Shea, B. J., Reeves, B. C., Wells, G., Thuku, M., Hamel, C., Moran, J., . . Henry, D. A. (2017). AMSTAR 2: a critical appraisal tool for systematic reviews that include randomised or nonrandomised studies of healthcare interventions, or both. BMJ, 358, j4008. https://doi.org/10.1136/bmj.j4008

Sheeran, P., Klein, W. M. P., \& Rothman, A. J. (2017). Health behavior change: Moving from observation to intervention. Annual Review of Psychology, 68, 573-600 https://doi.org/10.1146/annurev-psych-010416-044007

Sheeran, P., Maki, A., Montanaro, E., Avishai-Yitshak, A., Bryan, A., Klein, W. M. P., . . Rothman, A. J. (2016). The impact of changing attitudes, norms, and self-efficacy on health-related 
intentions and behavior: A meta-analysis. Health Psychology, 35, 1178-1188. https://doi.org/10.1037/hea0000387

Spring, B., Moller, A. C., \& Coons, M. J. (2012). Multiple health behaviours: Overview and implications. Journal of Public Health, 34, I3-I10. https://doi.org/10.1093/pubmed/fdr111

Suls, J., Mogavero, J. N., Falzon, L., Pescatello, L. S., Hennessy, E. A., \& Davidson, K. W. (2020). Health behaviour change in cardiovascular disease prevention and management: Meta-review of behavior change techniques to affect self-regulation. Health Psychology Review. https://doi.org/10.1080/17437199.2019.1691622

Teixeira, P. J., Marques, M. M., Silva, M. N., Brunet, J., Duda, J., Haerens, L., . . Hagger, M. S. (2020). Classification of techniques used in self-determination theory-based interventions in health contexts: An expert consensus study. Motivation Science. https://doi.org/10.31234/osf.io/z9wqu

West, R., Godinho, C. A., Bohlen, L. C., Carey, R. N., Hastings, J., Lefevre, C. E., \& Michie, S. (2019). Development of a formal system for representing behaviour-change theories. Nature Human Behaviour, 3, 526-536. https://doi.org/10.1038/s41562-019-0561-2

Wilson, T. E., Hennessy, E. A., Falzon, L., Boyd, R., Kronish, I. M., \& Birk, J. L. (2020). Effectiveness of interventions targeting self-regulation to improve adherence to chronic disease medications: A meta-review of meta-analyses. Health Psychology Review. https://doi.org/10.1080/17437199.2019.1706615

Zeng, X., Zhang, Y., Kwong, J. S. W., Zhang, C., Li, S., Sun, F., . . Du, L. (2015). The methodological quality assessment tools for preclinical and clinical studies, systematic review and meta-analysis, and clinical practice guideline: A systematic review. Journal of Evidence-Based Medicine, 8, 210. https://doi.org/doi:10.1111/jebm.12141

Zhang, C. Q., Zhang, R., Schwarzer, R., \& Hagger, M. S. (2019). A meta-analysis of the health action process approach. Health Psychology, 38, 623-637. https://doi.org/10.1037/hea0000728 
Figure 1. Illustration of a basic model of a behavior change mechanism of action (Hagger, 2019a). Figure 1a represents the mediation of a behavior change method or technique on behavior change through change in a modifiable theory-based factor along with the residual effect of the technique on behavior change. Figure $1 \mathrm{~b}$ represents the direct effect of the technique on behavior change, effectively illustrating intervention efficacy in the absence of putative mediators.

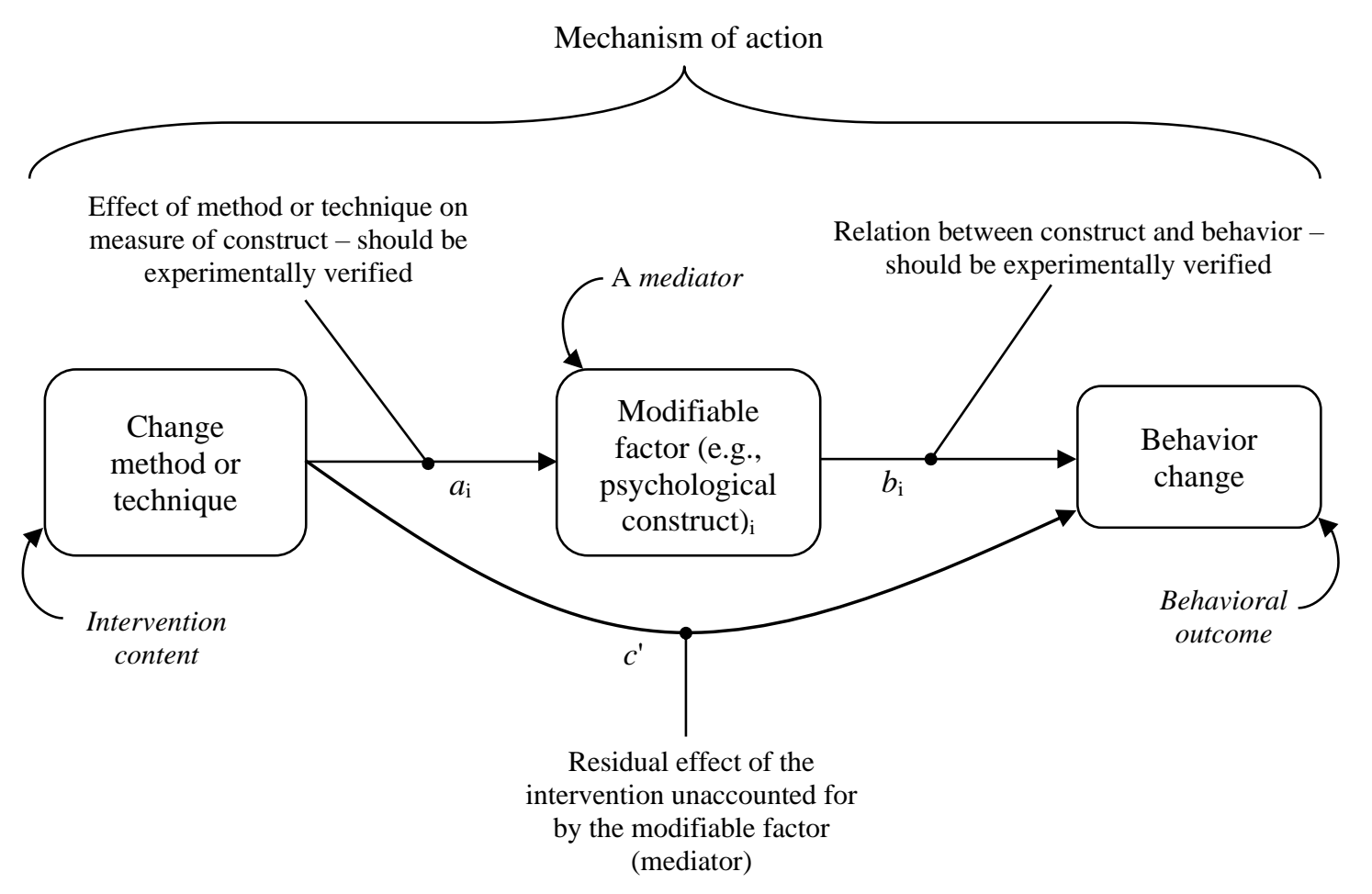

(1a)

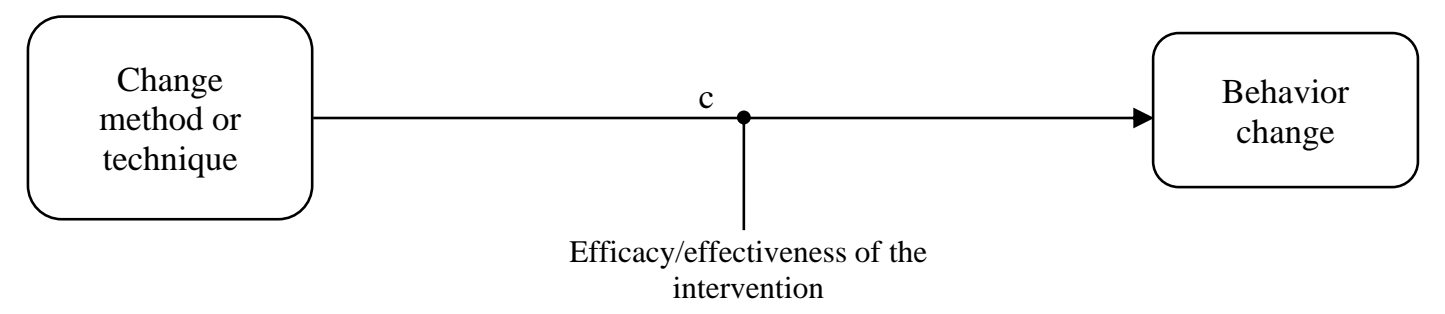

